\title{
6iniversity of Glasgow
}

Scaffardi, M., Andriolli, N., Malik, M. N., Zhang, N., Lazzeri, E., Klitis, C., Lavery, M., Sorel, M. and Bogoni, A. (2018) Multiplane Orbital Angular Momentum and Wavelength Switch based on Integrated Tunable Vortex Emitters. 2018 Optical Fiber Communication Conference, San Diego, CA, USA, 11-15 Mar 2018. Th2A.44. ISBN 9781943580385.

There may be differences between this version and the published version. You are advised to consult the publisher's version if you wish to cite from it.

http://eprints.gla.ac.uk/171644/

Deposited on: 22 October 2018

Enlighten - Research publications by members of the University of Glasgow http://eprints.gla.ac.uk 


\title{
Multiplane Orbital Angular Momentum and Wavelength Switch based on Integrated Tunable Vortex Emitters
}

\author{
M. Scaffardi ${ }^{(1)}$, N. Andriolli( ${ }^{(2)}$, M. N. Malik ${ }^{(1,2)}$, N. Zhang $^{(3)}$, E. Lazzeri ${ }^{(2)}$, C. Klitis ${ }^{(3)}$, M. Lavery ${ }^{(3)}$, \\ M. Sorel ${ }^{(3)}$, A. Bogoni ${ }^{(1,2)}$ \\ (1) CNIT, Via Moruzzi 1, 56124 Pisa, Italy - mirco.scaffardi@cnit.it \\ (2) Scuola Superiore Sant'Anna, Via Moruzzi 1, 56124 Pisa, Italy \\ (3) University of Glasgow, Oakfield Avenue, Glasgow G12 8LT, UK
}

\begin{abstract}
A multiplane switch architecture exploiting OAM and wavelength domains is characterized in terms of BER, scheduling/reconfiguration latency, and power consumption. Doubling the exploited OAM modes reduces the latency by $42 \%$ and the power by $17 \%$.

OCIS codes: (060.4259) Networks, packet-switched; 050.4865 Optical vortices.
\end{abstract}

\section{Introduction}

The continuous increase in the data center traffic imposes tight performance constraints to data center switches, in particular in terms of scalability and power consumption [1]. In this context, multiplane optical switch architectures are very attractive, since they enable large switch port counts in a modular way while complying with physical layer limitations. Space, wavelength, and time are typically exploited as switching domains in multiplane architectures [2]. The orbital angular momentum (OAM) has been recently introduced as a novel multiplexing and switching domain, guaranteeing high flexibility and scalability at low power consumption through innovative photonic integrated devices [3]. OAM relies on optical beams with azimuthal varying phase term: OAM of different order are orthogonal, i.e. they can co-propagate without interference. The use of OAM has been demonstrated for both free-space and fiber-based transmission, as well as for proof-of-concept switching (e.g., on $2 \times 2$ switch [3]). However no study has focused on the performance of OAM in large-scale switching architectures.

In this work we define for the first time a multiplane optical switch architecture exploiting both OAM and wavelength domains. In particular, the proposed architecture effectively exploits the recently proposed integrated OAM modulator/multiplexer based on tunable concentric vortex emitters [4], which converts multiple Gaussian beams at different wavelengths into OAM beams of selectable OAM order and also simultaneously multiplexes them. OAM modes are used to address the switch cards, while wavelengths are used to address the ports on each card. A specifically designed scheduler has been devised for the multiplane OAM-wavelength switch, adapting the two-step scheduling framework in [5] to the constraints arising from the OAM-wavelength technology. The proposed switch architecture has been experimentally characterized in terms of bit error rate (BER) and reconfiguration time for the different switch configurations, successfully demonstrating absence of penalty and fast switching $(10 \mu \mathrm{s})$. The devised scheduler has been assessed through simulations for different number of OAM modes and wavelengths. Finally, a power consumption analysis has been carried out, showing the inherent energy efficiency advantages of the proposed OAM-wavelength solution.

\section{Architecture description}

The proposed OAM-wavelength switch architecture is shown in Fig. 1, derived from space-based multiplane architectures [2] enhanced to exploit the integrated OAM modulator/multiplexer based on tunable concentric vortex emitters. The switch hosts $N$ input and output cards, each with $M$ electrical input and output ports ( $N M$ total switch ports). Cards are addressed by the OAM domain, while ports on a card are addressed by the wavelength domain. Aiming at energy efficiency, a single intercard laser array provides $M$ continuous-wave signals at different wavelengths, feeding $M$ Mach-Zehnder modulators (MZM) per card. All $M$ MZM electrical ports on each card are connected to a buffer stage responsible for queueing and routing the packets to the proper MZM according to the destination port. MZM outputs are connected to the $M$ inputs of the OAM modulator/multiplexer (OAM mod+mux, [6]), which converts each of the $M$ signals from Gaussian to an OAM of selectable order, and multiplexes them. The multiplexed OAM beams from the different cards, further multiplexed by means of common free-space beam combiners, propagate in free-space to reach a single OAM demodulator/demultiplexer (OAM demod+demux, realized with two cascaded patterned refractive elements followed by a lens [7]). The OAM demod+demux directs all beams carrying a specific OAM order to a given output card, irrespectively of the wavelength, and at the same time converts them to Gaussian. Each output is then amplified and sent to an arrayed waveguide grating (AWG) to separate the different wavelengths, each destined to a specific card output port. Each AWG output port is connected to an amplified photodiode (PD) to convert the signal into the electrical domain. 


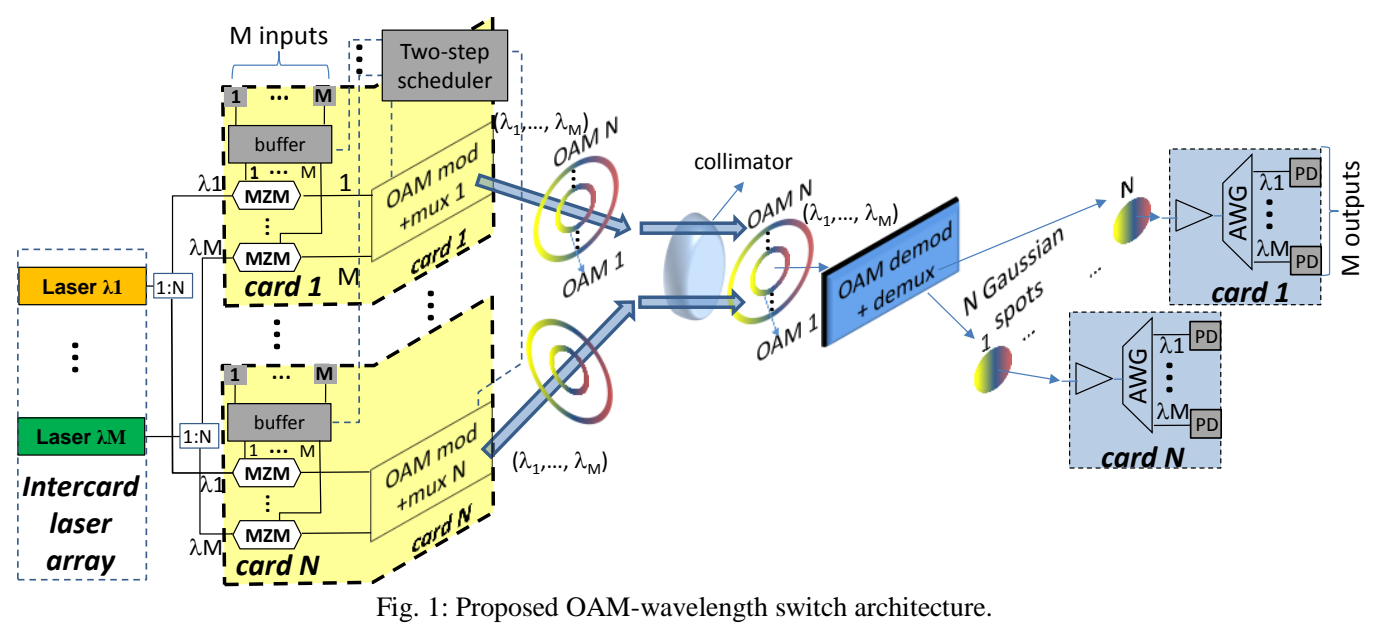

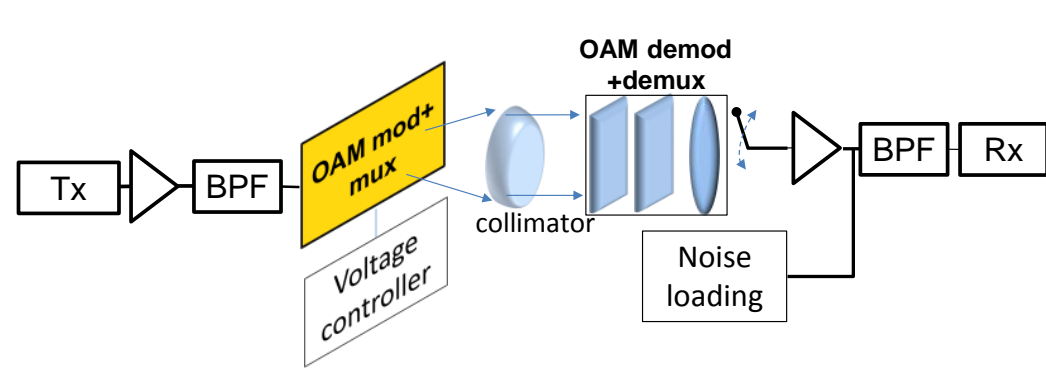

(a)

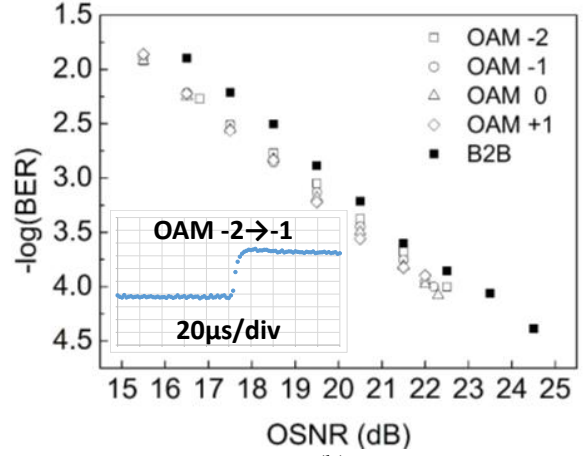

(b)

Fig. 2: (a) Experimental setup. Tx: transmitter; BPF: band pass filter; Rx: receiver. (b) BER vs. OSNR for different configurations of the integrated OAM mod+mux, emitting OAM modes with different order. Inset: output power transition for OAM mode switching $-2 \rightarrow-1$.

\section{Experimental and simulative analysis}

The feasibility of the architecture presented in Fig. 1 is experimentally investigated using the setup in Fig. 2(a). In order to test the switch in a demanding condition, a 30 Gbaud signal (16QAM) at $1547 \mathrm{~nm}$ is generated, amplified and sent to the OAM mod+mux [6], where it is converted from Gaussian to OAM. By changing the controller voltage, the waveguide refraction index in the OAM mod+mux is changed by means of the the thermo-optic effect, thus changing the order of the emitted OAM mode [8]. After the collimator and the OAM demod+demux (10 dB total loss of the OAM mux-demux cascade), the OAM demodulated and demultiplexed signal is coupled into a fiber by means of a lens and a nano-positioning system, and sent to the receiver chain. A noise-loading stage has been implemented to perform BER measurements as a function of the optical signal to noise ratio (OSNR), shown in Fig. 2(b). We tuned the OAM mod+mux over four different OAM modes $(-2,-1,0,+1)$, showing uniform BER among all of them, even slightly better than back-to-back due to the filtering effect of the OAM mod+mux acting as an adapted receiver [9]. A switch reconfiguration latency of $10 \mu \mathrm{s}$ has been measured for OAM transitions $-2 \rightarrow-1$ and $-2 \rightarrow+1$, the former being shown in the inset of Fig. 2(b).

This experimental validation shows that the OAM-based switch does not introduce any relevant OSNR penalty and requires a guard time of about $10 \mu \mathrm{s}$ : therefore in the following an impairment-unaware scheduling solution with packet durations in the order of $100 \mu$ s is considered.

A proper scheduler has been then devised, adapted from [5] to comply with the specific constraints of the OAM-wavelength multiplane architecture (namely, each wavelength cannot be simultaneously converted to multiple OAM modes on the same card) while being computationally efficient. As described in Fig. 3(a), a two-step scheduler is adopted, where the first step (intracard scheduling) is simultaneously executed on each card exploiting longest queue first (LQF) algorithm, and subsequently the second step (intercard scheduling) is simultaneously executed for each wavelength plane using iSLIP algorithm. Fixed-side packets with Bernoulli arrival process are synchronously switched every time slot, 1000-packet buffers per port are utilized. Fig. 3(b) shows the average packet latency as a function of the normalized network load for different card-port (i.e., $N \times M$ ) switch configurations summing up to 160 switch ports. The latency decreases for increasing number of supported OAM modes $N$ : among 
the tested configurations it is minimum for $N=10$, being up to $42 \%$ lower compared to $N=5$ at medium and high loads. Fig. 3(c) assesses the scalability of the switch scheduler, evaluating the average latency for different switch configurations using $N=10 \mathrm{OAM}$ modes and an increasing number of supported wavelengths $M$. The latency increase for increasing switch size is limited, confirming the good performance of the scheduler for the OAMwavelength architecture also in scaled-up configurations.

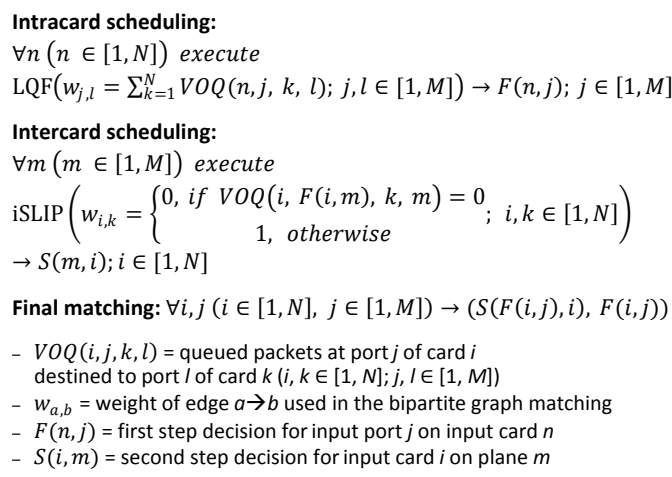

(a)

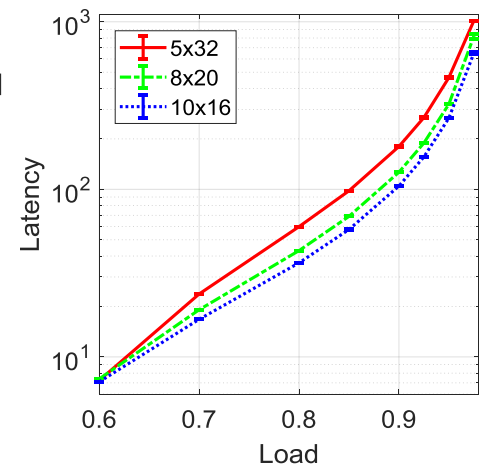

(b)

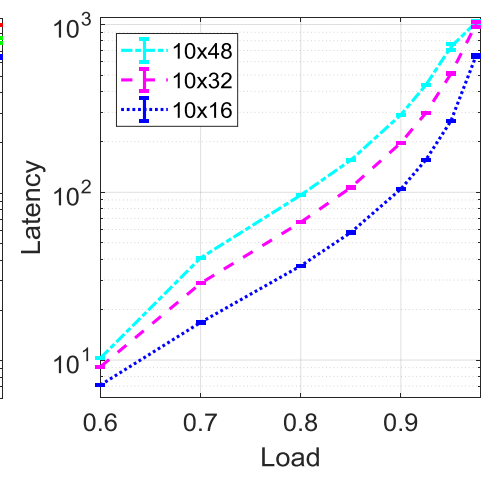

(c)

Fig. 3: (a) Operation of the two-step scheduling framework based on LQF and iSLIP. Average packet latency as a function of the normalized network load for different port/card configurations: (b) switch size $N M=160$ ports; (c) $N=10$ OAM modes and different wavelengths $M$.

The power consumption of the optical devices in the proposed switch (i.e., lasers, OAM multiplexers, optical amplifiers, photoreceivers) has been calculated for the same switch configurations as in Fig. 3(b) and 3(c): the results are summarized in Table 1. The power consumption decreases by increasing the number of OAM modes with respect to the number of wavelengths: in particular a 17\% decrease is observed when doubling the supported OAM modes in the 160-port switch, while the power increase is moderate for increasing number of wavelengths while keeping the OAM modes $N=10$. This is mainly due to the much lower power consumption of the OAM modulators/multiplexers ( $23 \mathrm{~mW}$ per OAM tuning step) compared to the lasers $(2400 \mathrm{~mW})$.

Table 1: Power consumption of the optical devices calculated for different combinations of number of OAM modes $N$ (cards) and number of wavelengths $M$ (ports per card).

\begin{tabular}{|c|c|c|c|c|c|}
\hline & \multicolumn{3}{|c|}{ Fixed number of switch ports } & \multicolumn{2}{c|}{ Fixed number of OAM modes } \\
\hline & $N=5, M=32$ & $N=8, M=20$ & $N=10, M=16$ & $N=10, M=32$ & $N=10, M=48$ \\
\hline $\begin{array}{c}\text { Power } \\
\text { consumption [W] }\end{array}$ & 144 & 123 & 120 & 157 & 196 \\
\hline
\end{tabular}

\section{Conclusions}

We proposed a multiplane switch architecture exploiting the OAM and wavelength domains. An implementation of the architecture based on integrated OAM modulators/multiplexers and on a refractive elements-based OAM demultiplexer/demodulator has demonstrated uniform performance in terms of BER without penalty and reconfiguration latency of $10 \mu \mathrm{s}$. A two-step scheduler has been specifically designed for the OAM-wavelength architecture and assessed through simulation. The utilization of a higher number of OAM modes is shown to be beneficial both in terms of scheduling latency and power dissipated by the optical devices.

This work has been funded by the EU H2020 Project ROAM (contract number: 645361). The authors acknowledge the support from the technical staff of the James Watt Nanofabrication Centre at the University of Glasgow.

\section{References}

[1] J. Shuja et al., "Survey of Techniques and Architectures for Designing Energy-Efficient Data Centers," IEEE Syst. J. 10, 507-519, (2016)

[2] O. Liboiron-Ladouceur et al., "A Scalable Space-Time Multi-Plane Optical Interconnection Network using Energy Efficient Enabling Technologies," IEEE/OSA J. Optic. Commun. and Netw. 3, A1-A11 (2011)

[3] M. Scaffardi et al., "A Silicon Microring Optical 2x2 Switch Exploiting Orbital Angular Momentum for Interconnection Networks up to 20Gbaud," IEEE/OSA J. Lightw. Tech. 35, 3142-3148 (2017)

[4] X. Cai et al., "Integrated compact optical vortex beam emitters," Science 338, 362-366 (2012)

[5] P. G. Raponi, et al., "Two-Step Scheduling Framework for Space-Wavelength Modular Optical Interconnection Networks," IET Commun. 4, 2155-2165 (2010)

[6] M. Scaffardi et al., "Tunable Orbital Angular Momentum (OAM) Conversion on 100Gb/s Real Data Traffic by Exploiting Concentric Waveguide Emitters," in Proc. ECOC'17, P2.SC5.4 (2017)

[7] M.P.J. Lavery et al., "Refractive elements for the measurement of the orbital angular momentum of a single photon," Opt. Express 20, 21102115 (2012)

[8] M.J. Strain et al., "Fast electrical switching of orbital angular momentum modes using ultra-compact integrated vortex emitters," Nature Comm. 5, 4856 (2014)

[9] F. Gambini et al., "Experimental demonstration of a 24-port packaged multi-microring network-on-chip in silicon photonic platform," Opt. Express 25, 22004-22016 (2017) 